\title{
Letter to the Editor in response to: Important knowledge for parents of children with heart disease: parent, nurse, and physician views
}

Keywords: Parental knowledge and understanding; families; psychosocial issues

Received: 18 January 2016; Accepted: 27 July 2016; First published online: 26 September 2016

\section{Knowledge and understanding for parents of children with heart disease}

We read the recent paper by Daily et $\mathrm{al}^{1}$ published in Cardiology in the Young with great interest because it focussed on identifying information that is important to parents of children with complex heart disease. We agree wholeheartedly that identification of areas of priority for information sharing with parents and the healthcare providers who care for them is most important. For parents having knowledge about the clinical signs to look for and knowing what to do, who to call, and where to go are crucial, because healthcare providers understand that ultimately parental care matters most.

We, however, find it interesting that the specific targeted parental education in this study did not include attention to the family's situation or issues that impact on parental knowledge and capacity building. We argue that identification of the knowledge needs of parents cannot be considered without attention to the needs and circumstances of the family in the first instance. How parents of children with serious health problems develop knowledge and act upon information depends on a range of issues that warrant mention. Some parents have better access to skills and resources than others. The range of issues that affect families can be complex and diverse - for instance, the impact of maternal emotional health on information processing and caregiving is well evidenced. ${ }^{2}$ The disruption to family functioning and decision making as a result of exposure to life stress is also well understood. ${ }^{3}$ Rather than parents understanding information, findings show that it is more a question of confidence. Research shows that mothers who feel anxious, or have little or no social support or someone to talk to, have lower levels of confidence in their knowledge and

Correspondence to: M. M. Tallon, School of Nursing, Midwifery and Paramedicine, Curtin University, Kent Street, Bentley, WA 6102, Australia. Tel: +61 089266 2053; Fax: +61 089266 4822; E-mail: M.Tallon@curtin.edu.au understanding about their children's heart disease. ${ }^{4}$ This is important because, in practice, paediatric healthcare providers are often concerned about how parents interpret and act upon important health information they are given about their children's care.

For parents, adjusting to the diagnosis of their child's complex CHD and managing the demands of complex feeding regimes and care can be consuming, and these challenges will be further compounded in the presence of additional psychosocial issues. The combination of managing their circumstances and the care needs of their children, while living with the worry about a guarded health outcome and the associated parental grief and loss, can be overwhelming. These families need substantial support to develop confidence in their knowledge and understanding, in order to create the best life possible for their affected child. By assessing and understanding the family's circumstances first, healthcare providers can identify and respond to the needs of the family, so that information that centres around pathophysiology and caregiving procedures can be tailored appropriately. In this way, assessment of the circumstances of the family could guide dissemination of knowledge and impact outcomes and child health.

\section{Mary M. Tallon, Garth E. Kendall School of Nursing, Midwifery and Paramedicine Curtin University, Bentley Western Australia, Perth, Australia}

Fiona Newall ${ }^{1,2}$

${ }^{1}$ Department of Nursing Research The Royal Children's Hospital Melbourne, Victoria, Australia

${ }^{2}$ Department of Nursing and Paediatrics The University of Melbourne Melbourne, Victoria, Australia 


\section{References}

1. Daily J, Fitzgerald M, Downing K, et al. Important knowledge for parents of children with heart disease: parent, nurse, and physician views. Cardiol Young 2016; 26: 61-69.

2. Berant E, Mikulincer M, Shaver P. Mothers' attachment style, their mental health, and their children's emotional vulnerabilities: a 7-year study of children with congenital heart disease. J Pers 2008; 76: 31-65.
3. McEwen B, Gianaros P. Central role of the brain in stress and adaptation: links to socioeconomic status, health, and disease (Report). Ann N Y Acad Sci 2010; 1186: 190-222.

4. Tallon M, Kendall G, Snider P. Development of a measure for maternal confidence in knowledge and understanding and examination of psychosocial influences at the time of a child's heart surgery. J Spec Pediatr Nurs 2015; 20: 36-48. 